\title{
Evaluation of the Three Glucometer Devices Performance in Comparison with the Cobas Integra 400 Plus Autoanalyzer in Measuring Blood Glucose Levels: A Comparative Cross-Sectional Study
}

\author{
Lotfi S. Bin Dahman ${ }^{*}{ }^{(0)}$, Ahmed M. Daakeek², Hussein S. Alghazali², \\ Abdullah M. Kaity ${ }^{3}$, Munir S. Obbed ${ }^{4}$ \\ ${ }^{1}$ Department of Medical Biochemistry, College of Medicine and Health Sciences, Hadhramout University, Mukalla, Yemen \\ ${ }^{2}$ Department of Internal Medicine, College of Medicine and Health Sciences, Hadhramout University, Mukalla, Yemen \\ ${ }^{3}$ Department of Community Medicine and Public Health, College of Nurse, Hadhramout University, Mukalla, Yemen \\ ${ }^{4}$ Department of Chemistry, College of Science, Hadhramout University, Mukalla, Yemen \\ Email: *lotfydahman@hu.edu.ye
}

How to cite this paper: Dahman, L.S.B., Daakeek, A.M., Alghazali, H.S., Kaity, A.M. and Obbed, M.S. (2021) Evaluation of the Three Glucometer Devices Performance in Comparison with the Cobas Integra 400 Plus Autoanalyzer in Measuring Blood Glucose Levels: A Comparative Cross-Sectional Study. Journal of Diabetes Mellitus, 11, 132-142. https://doi.org/10.4236/jdm.2021.114010

Received: June 27, 2021

Accepted: October 23, 2021

Published: October 26, 2021

Copyright $\odot 2021$ by author(s) and Scientific Research Publishing Inc. This work is licensed under the Creative Commons Attribution International License (CC BY 4.0).

http://creativecommons.org/licenses/by/4.0/

\begin{abstract}
Objective: The study aimed to evaluate the performance of the three glucometers compared to standard the laboratory method (Cobas Integra 400 Plus) in measuring blood glucose levels. Patients and Methods: A total of $100 \mathrm{Ye}-$ meni diabetic patients were randomly recruited into a comparative crosssectional study. Venous and finger-pricked blood samples were obtained from all participants and used for blood glucose levels measurement following the standard procedures. Results: The mean blood glucose levels for one-hundred diabetic patients using the Gluco Contour TS and Gluco SD Codefree were not significantly different compared with the Cobas Integra 400 Plus $(12.14 \pm 6.89 \mathrm{mmol} / \mathrm{L}$ vs. $12.85 \pm 8.83 \mathrm{mmol} / \mathrm{L}, P=0.159 ; 12.50 \pm$ $7.18 \mathrm{mmol} / \mathrm{L}$ vs. $12.85 \pm 8.83 \mathrm{mmol} / \mathrm{L}, P=0.490)$, respectively. However, there is a significant difference using the Gluco Alert device from that of the Cobas Integra 400 Plus $(11.83 \pm 6.94 \mathrm{mmol} / \mathrm{L}$ vs. $12.85 \pm 8.83 \mathrm{mmol} / \mathrm{L}, P=$ 0.046). Furthermore, using the ROC curve at a $95 \%$ confidence interval, the Cobas Integra 400 Plus showed a significant agreement with the Gluco Contour TS (51.4\%), Gluco SD Codefree (50.4\%), and Gluco Alert (39.3\%), respectively. For determining accuracy, the sensitivity of the glucometer devices was the following: Gluco SD Codefree (87.3\%), Contour TS (85.9\%), and Gluco Alert (78.9\%). In this regard, the highest specificity was related to Gluco Contour TS (65.5\%). Conclusion: The correlation between both me-
\end{abstract}


thods was good, with high sensitivity and specificity in measuring blood glucose levels as indicated by the ROC curve. Thus, we suggest using these glucometers at homes and hospitals as a point of care for diabetic patients.

\section{Keywords}

Diabetes Mellitus, Blood Glucose Levels, Glucometers, Cobas Integra 400

Plus Autoanalyzer

\section{Introduction}

Diabetes mellitus is one of the chronic metabolic disorders characterized by hyperglycemia resulting from either defect in insulin secretion, insulin action, or both [1]. Over 285 million people had diabetes in 2010 and are expected to reach 347 million in 2030 [2]. Retinopathy, nephropathy, neuropathy, and cardiovascular disorders as complications of diabetes may cause premature death in diabetic patients. Furthermore, these complications would impose additional costs on the family, community, and health care system [3]. Blood glucose level monitoring is being recognized as a priority in treating critically ill diabetic patients as it has helped reduce complications of diabetes [4] [5].

Today, regarding the importance of diabetes care management, the researchers have suggested "Self-Monitoring of Blood Glucose" (SMBG) to reduce the burden and increase cost-effectiveness [6] [7]. SMBG is a process of blood glucose checking by the patient known as a glucometer device. More than 44 million tests are performed daily worldwide, at a global cost of $>\$ 8.8$ billion per year [8]. Glucometers are devices designed to measure the glucose concentration of capillary blood obtained through a finger or heel puncture using a sterile lancet. According to the American Diabetes Association (ADA), SMBG is commonly applied three times a day [9].

The awareness of diabetic patients about the advantages of SMBG is rising. However, the glucometer devices available in the market have some issues regarding their standardization and their credibility to check blood glucose. The advantages of the glucometers include; small blood samples, simple utilization, and cost-effectiveness. However, the precision and accuracy of the devices are doubtful [10]. In this regard, the Center for Disease Control and prevention (CDC) has determined an expected error of $\pm 20 \mathrm{mg} / \mathrm{dl}$ for blood glucose $<100$ $\mathrm{mg}$ and $\pm 20 \%$ for blood glucose $>100 \mathrm{mg}$ [10]. Accordingly, some of the glucometer devices overestimate the blood glucose values; this makes it difficult to make the best clinical decision by health care workers [11].

Regarding the popularity of glucometer devices since 1980, many researchers demonstrated various credibility for these devices [12]; in this regard, some shortcomings have been raised about glucometer accuracy and precision in the fourth International Congress of SMBG application in 2011 [13]. The accuracy and precision are affected by many factors, e.g., sampling faults, calibration, 
body temperature, vasodepressor medications, edema, and hematocrit [14] [15].

Currently, in Yemen, however, no data is available on the accuracy and precision of these devices; we selected three common glucometers that have flooded the Yemeni market. These devices are Gluco Contour TS, Gluco SD Codefree, and Gluco Alert. Thus, the study aimed to evaluate the performance of the mentioned glucometers compared to the laboratory standard method (Cobas Integra 400 Plus) in measuring blood glucose levels using blood samples taken from diabetic patients at the Ibn-Sina Hospital in Mukalla, Yemen.

\section{Patients and Methods}

\subsection{Study Design and Population}

A total of one hundred Yemeni Type two diabetic patients (35 males and 65 females) were randomly recruited into a comparative cross-sectional study. The study was carried out at the National Center for the Public Health Laboratories in Mukalla, Yemen. According to Cochran's sample size determination formula, the sample size was calculated using the prevalence of diabetes (6.57\%) at a $95 \%$ confidence interval [16]. We then added $10 \%$ to avoid sample bias. Diabetic patients were diagnosed based on medical history, regular anti-diabetic medications, or the American Diabetes Association (ADA) criteria [1]. Inclusion criteria of the study were conscious, willing to participate in the study. Older than 18 years overnight fasting for at least eight hours having normal hematocrit, normal serum total cholesterol based on the previous history and laboratory examinations. In addition, patients with no coagulopathy disorders or taking vasoconstrictor agents such as adrenaline and dopamine were included in the study. The patients whose blood samples were hemolyzed were excluded. Patients were introduced to the study protocol, and they gave voluntary informed written consent. According to the Declaration of Helsinki, the study protocol was implemented and granted by the Ethics Committee of the Medicine College, Hadhramout University, Yemen.

\subsection{Data and Sample Collection}

The data collected using a self-administered pretested questionnaire was designed by Medical Laboratory Sciences, Medicine and Health Sciences College, Hadhramout University. The questionnaire focuses on socio-demographic data, the history of diabetic patients, and questions about glucometer device usage. Before data collection, the questionnaire is pretested. The questionnaire was conducted on ten patients to check the understanding and applicability of the questionnaire. Some linguistic modifications confuse problems based on the results, making them more effortless experiences and interpretations by the patients. Five milliliters of venous blood were taken from each participant after overnight fasting. The blood sample collected in tubes without anticoagulants then separated and stored at $-20^{\circ} \mathrm{C}$ till analyses. Besides, one drop of blood was taken from the capillary of fingers using a sterile lancet following standard procedures as described by the manufacturer's directions. Capillary and venous 
blood samples were collected by trained medical laboratory techniques following standard procedures.

\subsection{Instruments}

The glucometer devices used in the study are Gluco Contour TS (Japan), Gluco SD Codefree (Korea), and Gluco Alert (China). The glucometer devices are verified by the American Diabetes Association (ADA) and Committee Europe (CCE) organization guidelines. Blood glucose concentration was measured using the Cobas Integra 400 Plus autoanalyzer (Roche Diagnostics, Mannheim, Germany). The Cobas Integra 400 Plus autoanalyzer calibration was evaluated by checking the system using a solution, namely "control samples". Capillary and venous blood glucose levels were measured according to the standard procedures described by the manufacturer's instructions of the mentioned glucometer devices and Cobas Integra 400 Plus autoanalyzer.

\subsection{Statistical Analysis}

Data were analyzed using SPSS Statistical Package (version 24). The Shapiro test was used to analyze the normal distribution of the continuous variables. Paired sample T-test was applied to specify the precision and Kappa agreement coefficient to compare the agreement between the three glucometer devices. Its score is between -1 to +1 ; more score indicates more reliability. We also used ROC curve analysis to demonstrate the sensitivity and specificity to show the accuracy of these glucometers (the area under each ROC curve). $P$-value $<0.05$ was considered statistically significant at a $95 \%$ confidence interval.

\section{Results}

Overall, 100 patients, 35 are males, and 65 are females with a mean age of 45.39 \pm 7.56 (Table 1). The blood glucose levels of diabetic Yemeni patients enrolled in this study were measured using the three different glucometer devices versus the standard laboratory method (Cobas Integra 400 Plus) at the National Center for Public Health Laboratories of Mukalla, Yemen.

Our results didn't show any significant statistical difference between Gluco Contour TS and Gluco SD Codefree compared with Cobas Integra 400 Plus autoanalyzer $(12.14 \pm 6.89 \mathrm{mmol} / \mathrm{L}$ vs. $12.85 \pm 8.83 \mathrm{mmol} / \mathrm{L}, P=0.159 ; 12.50 \pm$ $7.18 \mathrm{mmol} / \mathrm{L}$ vs. $12.85 \pm 8.83 \mathrm{mmol} / \mathrm{L}, P=0.490)$ respectively. However, blood glucose levels using the Gluco Alert device was significantly lower than the Cobas Integra 400 Plus autoanalyzer $(11.83 \pm 6.94 \mathrm{mmol} / \mathrm{L}$ vs. $12.85 \pm 8.83$ $\mathrm{mmol} / \mathrm{L}, P=0.046$ ) (Table 2), indicates an acceptable precision of Gluco Contour TS and Gluco SD Codefree glucometers.

According to the results of Pearson's correlation coefficient, there was a positive correlation between the three glucometer devices compared to the Cobas Integra 400 Plus autoanalyzer (Gluco Contour TS; $\mathrm{r}=0.826, P<0.001$, Gluco SD Codefree; $\mathrm{r}=0.827, P<0.001$, and Gluco Alert; $\mathrm{r}=0.822, P<0.001$ ) (Figures $1(\mathrm{a})-(\mathrm{c}))$. 
Table 1. Baseline characteristics of the participants.

\begin{tabular}{|c|c|c|c|}
\hline No. (100) & Mean \pm SD & Frequency (n) & Percentage (\%) \\
\hline Age (years): & $45.39 \pm 7.56$ & & \\
\hline \multicolumn{4}{|c|}{ Age categories (years): } \\
\hline$\leq 40$ & & 36 & 36 \\
\hline $41-50$ & & 36 & 36 \\
\hline$\geq 51$ & & 28 & 28 \\
\hline \multicolumn{4}{|l|}{ Sex: } \\
\hline Male & & 35 & 35 \\
\hline Female & & 65 & 65 \\
\hline
\end{tabular}

Table 2. Comparison of the mean blood glucose levels between the glucometers and Cobas Integra 400 Plus autoanalyzer.

\begin{tabular}{|c|c|c|c|}
\hline & Mean $\pm S D$ & $\mathrm{~T}$ & $P$-value \\
\hline $\begin{array}{l}\text { Gluco Contour TS vs. } \\
\text { Cobas Integra } 400 \text { Plus }\end{array}$ & $12.14 \pm 6.89$ vs. $12.85 \pm 8.83$ & 1.41 & 0.159 \\
\hline $\begin{array}{l}\text { Gluco SD Codefree vs. } \\
\text { Cobas Integra } 400 \text { Plus }\end{array}$ & $12.50 \pm 7.18$ vs. $12.85 \pm 8.83$ & 0.69 & 0.490 \\
\hline $\begin{array}{c}\text { Gluco Alert vs. } \\
\text { Cobas Integra } 400 \text { Plus }\end{array}$ & $11.83 \pm 6.94$ vs. $12.85 \pm 8.83$ & 2.01 & 0.046 \\
\hline
\end{tabular}

Data are presented by mean \pm standard deviation (SD). Paired sample T-test was used to compare between the mean values of Cobas Integra 400 Plus autoanalyzer vs. Gluco Contour TS, Gluco SD Codefree and Gluco Alert devices at 95\% confidence interval and the differences were considered statistically significant if $P<0.05$.

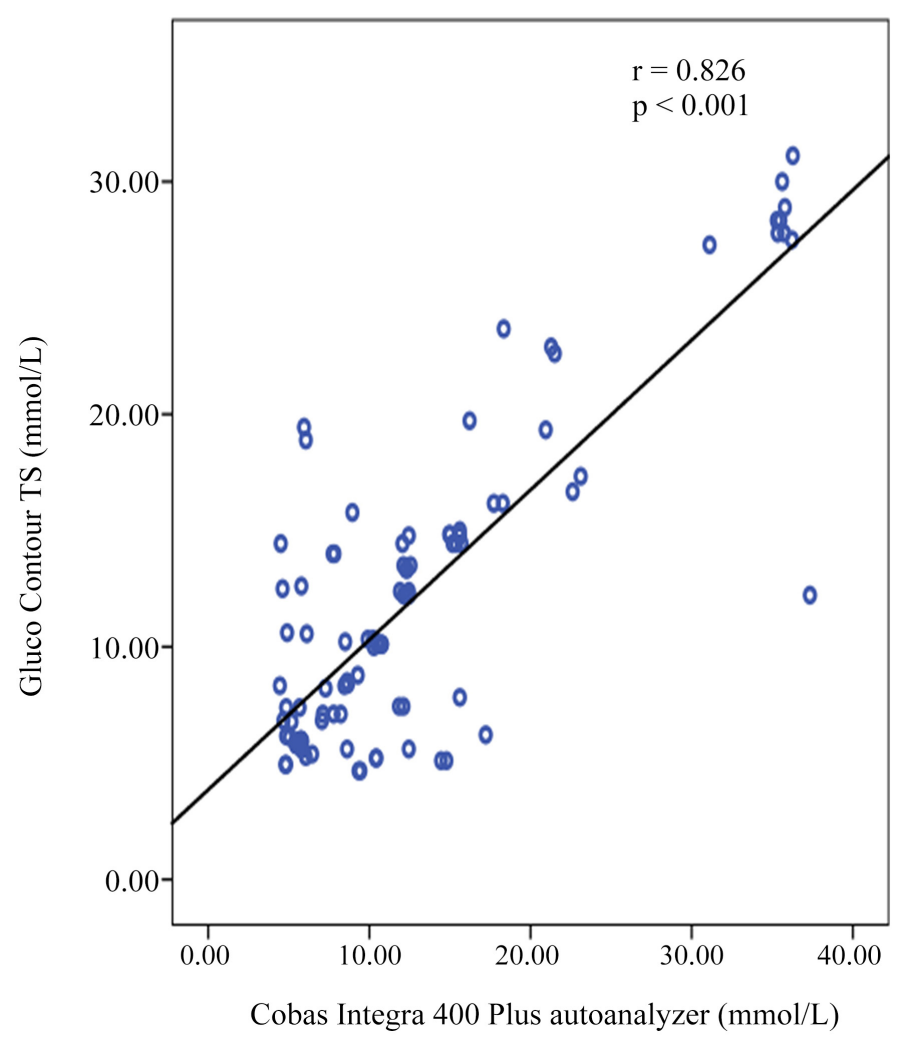

(a) 


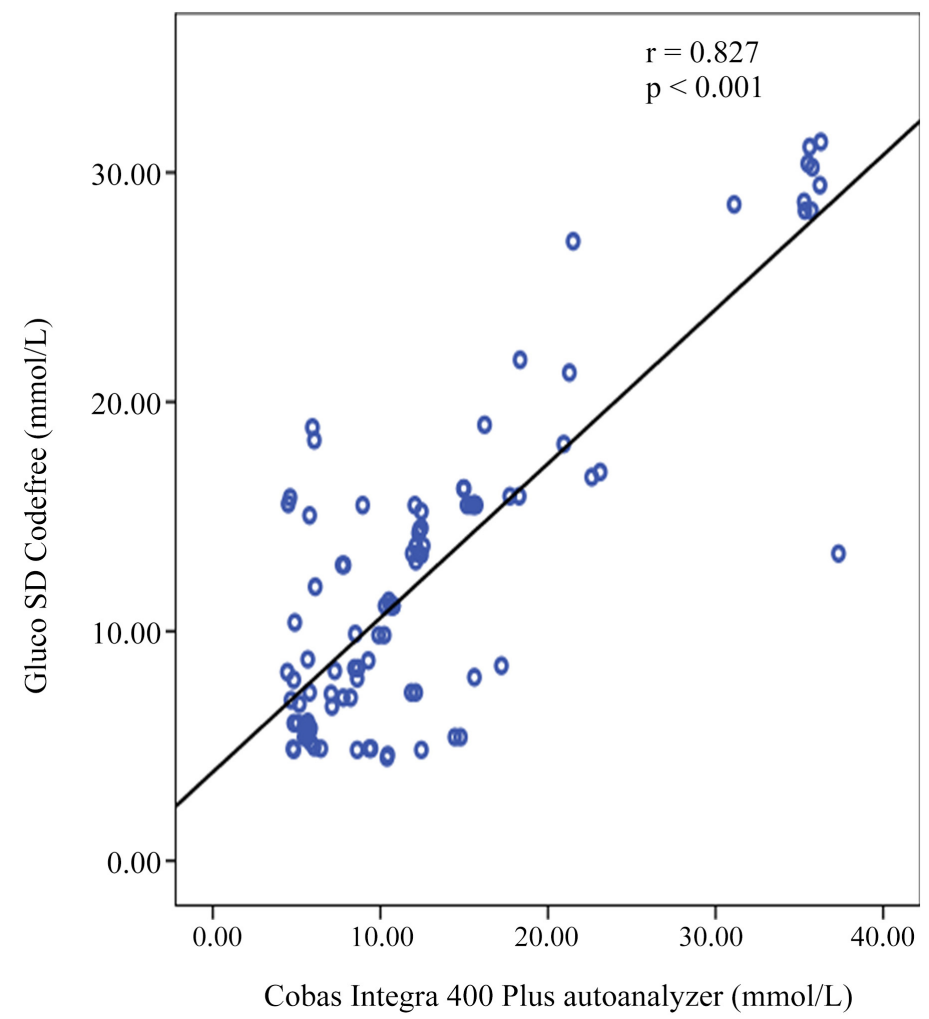

(b)

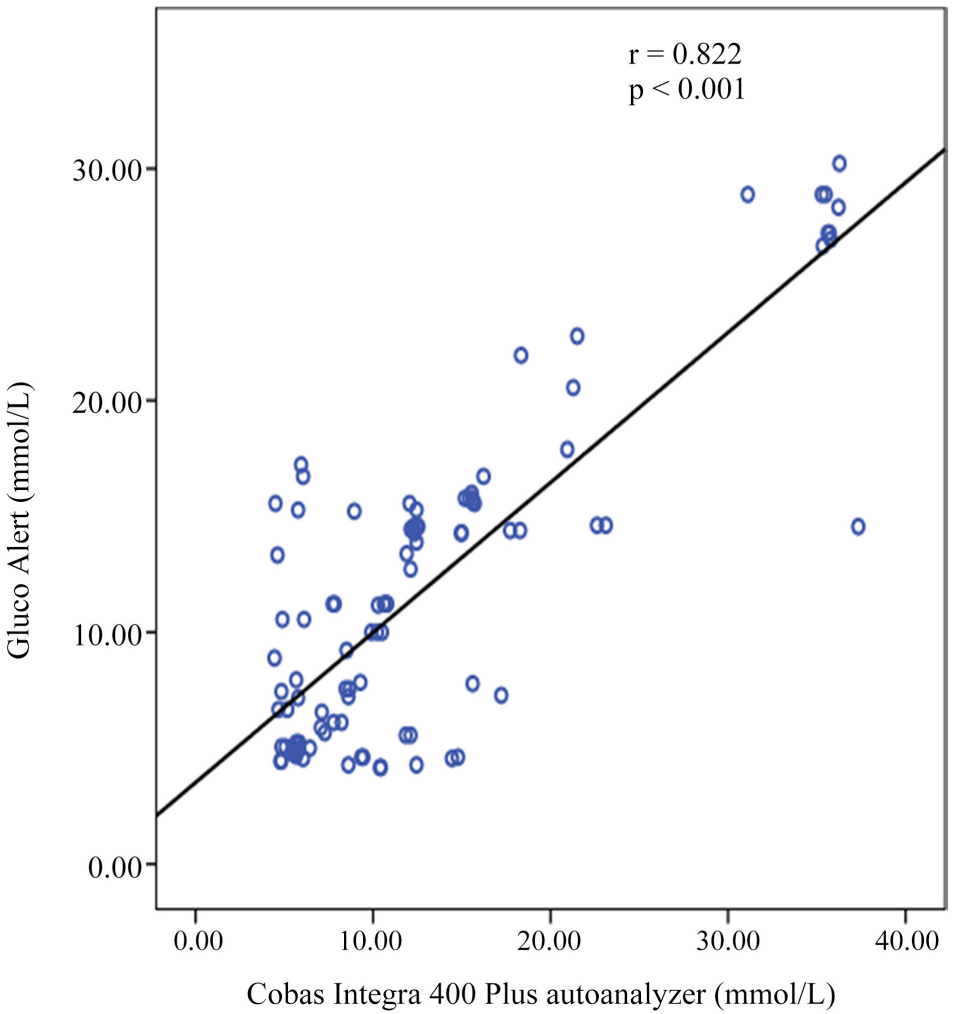

(c)

Figure 1. Correlation between blood glucose levels measured using glucometers and Cobas Integra 400 Plus autoanalyzer. 
Furthermore, ROC curve indicates the overall sensitivity and specificity of the three different glucometers compared with Cobas Integra 400 Plus autoanalyzer using ROC curve at 95\% confidence interval (Table 3 and Figure 2). Although the sample is small in size, the Cobas Integra 400 Plus autoanalyzer showed a significant agreement with Gluco Contour TS (51.4\%), Gluco SD Codefree (50.4\%), and Gluco Alert (39.3\%), respectively. For determine the accuracy of the glucometers, the sensitivity of Gluco SD Codefree (87.3\%), higher than Contour TS (85.9\%) and Gluco Alert (78.9\%), respectively. In this regard, the highest specificity was related to the Gluco Contour TS (65.5\%).

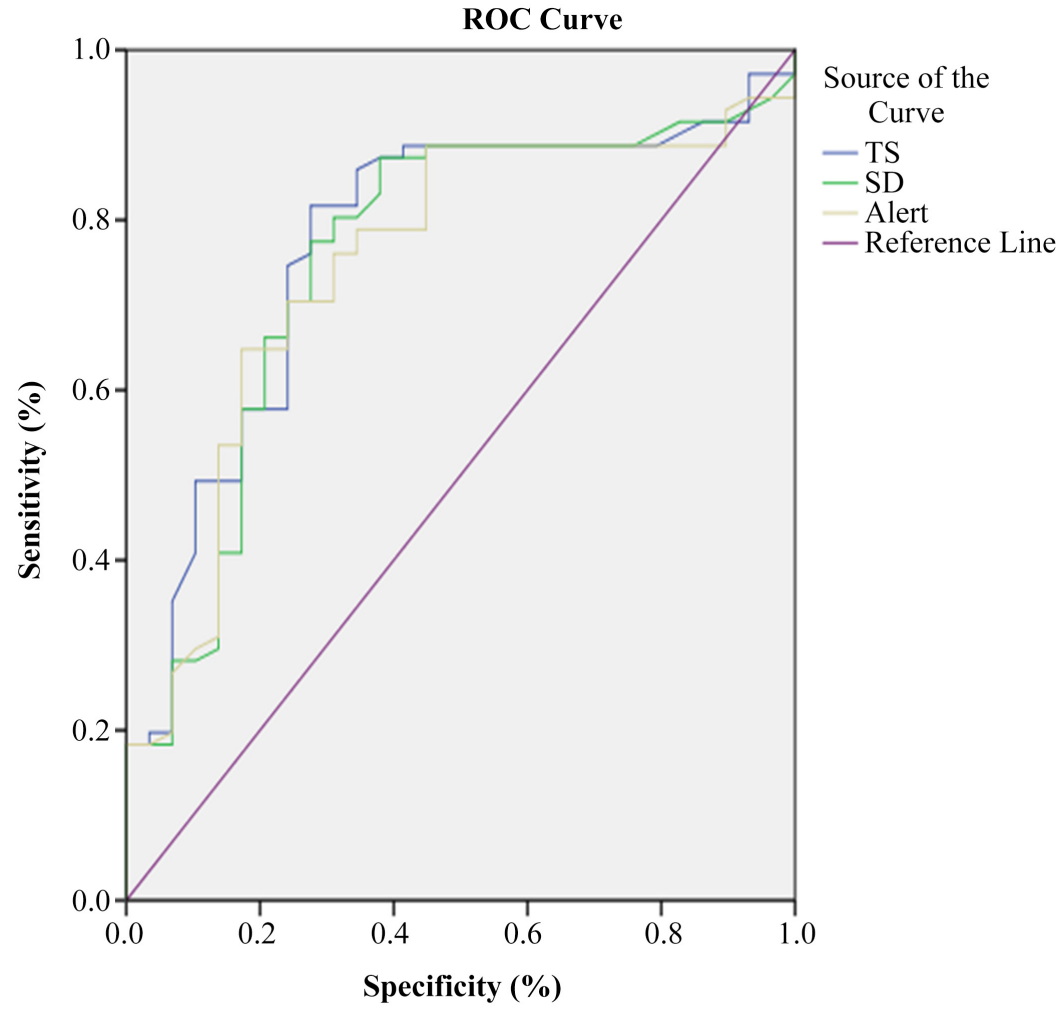

Diagonal segments are produced by ties.

Figure 2. The area under the curve by using ROC curve analysis.

Table 3. Sensitivity, specificity and accuracy of the glucometers in measuring blood glucose levels.

\begin{tabular}{cccccc}
\hline Glucometers & $\begin{array}{c}\text { Sensitivity } \\
(\%)\end{array}$ & $\begin{array}{c}\text { Specificity } \\
(\%)\end{array}$ & $\begin{array}{c}\text { Accuracy } \\
(\%)\end{array}$ & $\begin{array}{c}\text { Kappa } \\
\text { coefficient (\%) }\end{array}$ & \begin{tabular}{c}
$\boldsymbol{P}$-value \\
\hline Gluco Contour TS
\end{tabular} \\
Gluco SD Codefree & 87.9 & 65.5 & 77.1 & 51.4 & $<0.001$ \\
Gluco Alert & 78.9 & 62.1 & 75.4 & 50.4 & $<0.001$ \\
\hline
\end{tabular}

ROC curve indicates the overall sensitivity and specificity of three different glucometer devices in comparison with the Cobas Integra 400 Plus autoanalyzer using ROC curve at 95\% confidence interval. The differences were considered statistically significant if $P<$ 0.05 . 


\section{Discussion}

Self-monitoring of blood glucose (SMBG) using glucometer devices as an essential part of diabetes care is increasing because it is an easy to perform and cost-effective approach [6]. However, conflicting data have been observed concerning the accuracy of these devices [17] [18] [19]. Thus, the current study was performed to evaluate the performance of three different glucometers (Gluco Contour TS, Gluco SD Codefree, and Gluco Alert) by comparing them with the standard laboratory method (Cobas Integra 400 Plus) in measuring blood glucose levels at the National Center for the Public Health Laboratories in Mukalla, Yemen.

Previous studies reported that no statistically significant irrespective of the diabetes status [20] [21] [22] [23], but they agree with the current study. However, other studies reported that the glucometer generally produced lower glucose readings than the standard laboratory method [24] [25]. Although the glucometer devices and the standards for comparisons in these studies are different, the principles of the test are the same. They, therefore, make our results are comparable to the findings of these studies.

Blood glucose levels in the capillary differ from those in the veins. Venous plasma glucose level is the estimated glucose after utilization of glucose by tissues. It depends on the extent of tissue extraction of glucose and the effect of insulin and insulin counterregulatory hormones [21]. In addition, the difference in blood glucose levels recorded by the glucometers and the standard laboratory method may be accounted for partly by many factors such as sampling faults, calibration, body temperature, vasodepressor medications, edema, and hematocrit [14] [15] [19].

Similarly, many investigators in the Intensive Care Evaluation and Survival Using Glucose Algorithm Regulation (NICE-SUGAR) study found that the specific lots of the glucometer strips varied considerably in their susceptibility to loss accuracy because of variation in hematocrit. They hypothesized that some of the true blood glucose levels in the hypoglycemic range might have been missed because of falsely elevated glucometer readings [26].

Moreover, estimation of blood glucose levels by glucometers may give outliers values which may cause medical errors by patients, their family members, or their care providers. Unfortunately, the International Organization for Standardization (ISO) and U.S. Food and Drug Administration (FDA) allows up to 5\% of values obtained by the glucometers to be outliers of any degree of magnitude [27]. However, our results showed a good correlation between the glucometers and the Cobas Integra 400 Plus autoanalyzer. Thus, the glucometers used in this study are relatively accurate in measuring blood glucose levels irrespective status of diabetic patients. These findings agree with some studies conducted by [23] [28], who found similar levels of correlation between the glucometer and the standard autoanalyzer. In contrast, a study conducted by [29] found a weak correlation between both methods in determining neonatal hypoglycemia. 
A rather exciting finding observed in this study is that the ROC curve showed the glucometers to be an even better method than the autoanalyzer indicated by the area under the curve because the area under the ROC curve represents a measure of discrimination to classify correctly those with and without the disease. Overall, the above findings indicated that the glucometers can still be used for SMBG as a point of care for diabetic patients; the standardization of these glucometers be given attention in policies geared toward diabetes care.

\section{Conclusion}

The current study found the correlation between glucometer and standard laboratory methods, with high sensitivity and specificity in measuring blood glucose levels as indicated by the ROC curve analysis. However, further studies with larger samples are recommended to confirm the reliability of such glucometers as a point of care for diabetic patients.

\section{Data Availability}

All requests for data access should be addressed to the corresponding author. Proposals requesting data access will have to specify how they plan to use the data.

\section{Acknowledgements}

The authors are grateful to the National Center for the Public Health Laboratories in Mukalla, Yemen, for funding and technical support. Also, special thanks to Students of Medical Laboratory Sciences Department (Abdulla Baamoor, Ahmed Ba Mokhtar, and Mohammed Alsomhi) for data collection and analysis performance.

\section{Conflicts of Interest}

The authors declare no conflicts of interest regarding the publication of this paper.

\section{References}

[1] American Diabetes Association (2014) Diagnosis and Classification of Diabetes Mellitus. Diabetes Care, 37, S81-S90. https://doi.org/10.2337/dc14-S081

[2] Zhang, P., Zhang, X., Brown, J., Vistisen, D., Sicree, R., Shaw, J., et al. (2010) Global Healthcare Expenditure on Diabetes for 2010 and 2030. Diabetes Research and Clinical Practice, 87, 293-301. https://doi.org/10.1016/j.diabres.2010.01.026

[3] American Diabetes Association (2008) Economic Costs of Diabetes in the US in 2007. Diabetes Care, 31, 596-615. https://doi.org/10.2337/dc08-9017

[4] Van den Berghe, G., Wouters, P.J., Bouillon, R., Weekers, F., Verwaest, P., Schetz, M., et al. (2003) Outcome Benefit of Intensive Insulin Therapy in the Critically Ill: Insulin Dose Versus Glycemic Control. Critical Care Medicine, 31, 359-366. https://doi.org/10.1097/01.CCM.0000045568.12881.10

[5] Van den Berghe, G., Wilmer, A., Hermans, G., Meersseman, W., Wouters, P.J., Mi- 
lants, I., et al. (2006) Intensive Insulin Therapy in the Medical ICU. The New England Journal of Medicine, 354, 449-461. https://doi.org/10.1056/NEJMoa052521

[6] Kalateh Jari, M., Sohrabi, M.B., Khosravi, A. and Zou Alfaghari, P. (2008) Correlation between Blood Glucose Measured Using Glucometers and Standard Laboratory Methods. Iranian Journal of Endocrinology and Metabolism, 10, 277-283.

[7] Lagarde, W.H., Barrows, F.P., Davenport, M.L., Kang, M., Guess, H.A. and Calikoglu, A.S. (2006) Continuous Subcutaneous Glucose Monitoring in Children with Type 1 Diabetes Mellitus: A Single-Blind, Randomized, Controlled Trial. Pediatric Diabetes, 7, 159-164. https://doi.org/10.1111/j.1399-543X.2006.00162.x

[8] Scott, M.G. (2010) Glucose Meters: Need for Greater Accuracy (Lab Perspective). In: Joint ADA/AACE Symposium-Point of Care Devices for Glucose and HbA1C: Are They Up to the Task? 70th Annual ADA Scientific Sessions, Orlando, 28 June 2010.

[9] American Diabetes Association (2016) Standards of Medical Care in Diabetes-2016 Abridged for Primary Care Providers. Clinical Diabetes: A Publication of the American Diabetes Association, 34, 3. https://doi.org/10.2337/diaclin.34.1.3

[10] Freckmann, G., Baumstark, A., Jendrike, N., Zschornack, E., Kocher, S., Tshiananga, J., et al. (2010) System Accuracy Evaluation of 27 Blood Glucose Monitoring Systems According to DIN EN ISO 15197. Diabetes Technology \& Therapeutics, 12, 221-231. https://doi.org/10.1089/dia.2009.0128

[11] Van den Berghe, G., Wouters, P., Weekers, F., Verwaest, C., Bruyninckx, F., Schetz, M., et al. (2001) Intensive Insulin Therapy in Critically Ill Patients. New England Journal of Medicine, 345, 1359-1367. https://doi.org/10.1056/NEJMoa011300

[12] Mann, E.A., Pidcoke, H.F., Salinas, J., Wade, C.E., Holcomb, J.B. and Wolf, S.E. (2007) Accuracy of Glucometers Should Not Be Assumed. American Journal of Critical Care, 16, 531-532. https://doi.org/10.4037/ajcc2007.16.6.531-a

[13] Mankovsky, B.N. (2011) 4th Annual Symposium on Self-Monitoring of Blood Glucose (SMBG) Applications and Beyond, May 12-14, 2011, Budapest, Hungary. Diabetes Technology \& Therapeutics, 13, 1161-1176.

https://doi.org/10.1089/dia.2011.0200

[14] Hortensius, J., Slingerland, R.J., Kleefstra, N., Logtenberg, S.J., Groenier, K.H., Houweling, S.T., et al. (2011) Self-Monitoring of Blood Glucose: The Use of the First or the Second Drop of Blood. Diabetes Care, 34, 556-560. https://doi.org/10.2337/dc10-1694

[15] Karon, S., Griesmann, L., Scott, R., Bryant, S.C., Dubois, J.A., Shirey, T.L., et al. (2008) Evaluation of the Impact of Haematocrit and Other Interference on the Accuracy of Hospital-Based Glucose Meters. Diabetes Technology \& Therapeutics, 10, 111-1120. https://doi.org/10.1089/dia.2007.0257

[16] Gunaid, A.A. (2002) Prevalence of Known Diabetes and Hypertension in the Republic of Yemen. Eastern Mediterranean Health Journal, 8, 374-385.

[17] Salacinski, A.J., Alford, M., Drevets, K., Hart, S. and Hunt, B.E. (2014) Validity and Reliability of a Glucometer against Industry Reference Standards. Journal of Diabetes Science and Technology, 8, 95-99. https://doi.org/10.1177/1932296813514315

[18] Rajendran, R. and Rayman, G. (2014) Point-of-Care Blood Glucose Testing for Diabetes Care in Hospitalized Patients an Evidence-Based Review. Journal of Diabetes Science and Technology, 8, 1081-1090. https://doi.org/10.1177/1932296814538940

[19] Ginsberg, B.H. (2009) Factors Affecting Blood Glucose Monitoring: Sources of Errors in Measurement. Journal of Diabetes Science and Technology, 3, 903-913. https://doi.org/10.1177/193229680900300438 
[20] Shete, S., Khan, H., Siddiqui, A.M. and Shinde, A. (2016) A Comparative Study of Venous and Capillary Blood Glucose Levels by Semi Autoanalyser and Glucometer. International Journal of Recent Trends in Science and Technology, 1, 46-48.

[21] Patel, N. and Patel, K. (2015) A Comparative Study of Venous and Capillary Blood Glucose Levels by Different Method. GCSMC Journal of Medical Sciences, 4, 53-56.

[22] Baig, A., Siddiqui, I., Jabbar, A., Azam, I.S., Sabir, S., Alam, S. and Ghani, F. (2007) Comparison between Bedside Testing of Blood Glucose by Glucometer vs. Centralized Testing in Tertiary Health Care Hospital. Journal of Ayub Medical College Abbottabad, 19, 25-29.

[23] Kanwugu, O.N., Helegbe, G.K., Aryee, P.A., Akontatiba, N.A, et al. (2017) A Comparative Assessment of the Glucose Monitor (SD Codefree) and Autoanalyzer (BT-3000) in Measuring Blood Glucose among Diabetic Patients. BMC Research Notes, 10, 453. https://doi.org/10.1186/s13104-017-2789-0

[24] Sudan, K. (2014) Comparison between Glucometer and Chemical Analyzer for Measuring Blood Glucose of Diabetic Patients. International Journal of Current Research, 6, 6610-6613.

[25] Bimenya, G.S., Nzarubara, G.R., Kiconco, J., Sabuni, S. and Byarugaba, W. (2003) The Accuracy of Self-Monitoring Blood Glucose Meter Systems in Kampala Uganda. African Health Sciences, 3, 23-32.

[26] Cembrowski, G.S., Tran, D.V., Slater-Maclean, L., Chin, D., Gibney, R.T.N. and Jacka, M. (2010) Could Susceptibility to Low Hematocrit Interference Have Compromised the Results of the NICESUGAR Trial? Clinical Chemistry, 56, 1193-1195. https://doi.org/10.1373/clinchem.2010.146217

[27] Krouwer, J.S. and Cembrowski, G.S. (2010) A Review of Standards and Statistics Used to Describe Blood Glucose Monitor Performance. Journal of Diabetes Science and Technology, 4, 75-83. https://doi.org/10.1177/193229681000400110

[28] Corstjens, A.M., Ligtenberg, J.J., van der Horst, I.C., Spanjersberg, R., Lind, J.S., et al. (2006) Accuracy and Feasibility of Point-of-Care and Continuous Blood Glucose Analysis in Critically ill ICU Patients. Critical Care, 10, R135. https://doi.org/10.1186/cc5048

[29] MostafaGharehbaghi, M. and Ghergherehchi, R. (2016) Comparison of Diagnostic Value of Blood Glucose Meter with that of Laboratory Method in Neonatal Hypoglycemia. Journal of Guilan University of Medical Sciences, 25, 106-112. 